


\title{
DEVELOPMENT AND LAB-SCALE TESTING OF A GAS GENERATOR HYBRID FUEL IN SUPPORT OF THE HYDROGEN PEROXIDE HYBRID UPPER STAGE PROGRAM
}

\author{
Gary K. Lund, Wm. David Starrett and Kent C. Jensen \\ ATK, Thiokol Propulsion Corp. \\ Brigham City, Utah
}

\begin{abstract}
$\underline{\text { Abstract }}$
As part of a NASA funded contract to develop and demonstrate a gas generator cycle hybrid rocket motor for upper stage space motor applications, the development and demonstration of a low sensitivity, high performance fuel composition was undertaken. The ultimate goal of the development program was to demonstrate successful hybrid operation (start, stop, throttling) of the fuel with high concentration $(90+\%)$ hydrogen peroxide. The formulation development and lab-scale testing of a simple DOT Class $1.4 \mathrm{c}$ gas generator propellant is described.
\end{abstract}

Both forward injected center perforated and aft injected end burner hybrid combustion behavior were evaluated with gaseous oxygen and catalytically decomposed $90 \%$ hydrogen peroxide. Cross flow and static environments were found to yield profoundly different combustion behaviors, which were further governed by binder type, oxidizer level and, significantly, oxidizer particle size.

Primary extinguishment was accomplished via manipulation of PDL behavior and oxidizer turndown, which is enhanced with the hydrogen peroxide system. Laboratory scale combustor results compared very well with 11 -inch and 24 -inch sub-scale test results with $90 \%$ hydrogen peroxide.

\section{Introduction}

Growing international competition for delivery of orbital payloads has prompted increased government and industry investment in advanced propulsion systems. NASA is funding efforts to investigate peroxide based propulsion systems, including hybrids.
Hybrid rockets have long been considered a low cost alternative due to their inherent safety, storability, and operational efficiency. Combining these features with the clean burning, storable, non-toxic attributes of hydrogen peroxide yields an excellent combination.

NASA has recently funded an effort to develop a peroxide hybrid upper stage propulsion system. The team, which is comprised of Lockheed Martin, Thiokol, and Rocketdyne, seeks to design, integrate, and test a peroxide thrust chamber assembly that will be capable of delivering 10,000 pounds of thrust at a vacuum specific impulse of 310 seconds for approximately 360 seconds.

The candidate system chosen for investigation is a gas generator design incorporating a partially oxidized, fuel-rich propellant with combustion initiated by, and made more efficient with, the injection of high concentration hydrogen peroxide. Multiple starts, steady state operation, and extinguishment of the hybrid motors are planned as part of this investigation. This paper will focus on the development, lab-scale testing/evaluation, and selection of the fuel formulation for this system.

\section{Technical Approach}

The fuel requirements for the gas generator cycle hybrid are challenging from a formulation perspective. Specifically, the fuel is to function as a propellant with respect to combustion characteristics (i.e. exhibit pressure dominated burn rate), yet readily extinguish upon oxidizer flow termination. Fuel formulation goals for this program were as follows:

Copyright 2001 ATK, Thiokol Propulsion Corp.

Published by the American Institute of

Aeronautics and Astronautics, Inc., with permission. 
1) Vacuum specific impulse with $90 \%$ hydrogen peroxide $\left(\mathrm{H}_{2} \mathrm{O}_{2}\right), 200$ psia chamber pressure, $100 / 1$ expansion ratio: $>\mathbf{3 1 0}$ seconds.

2) A vailable, low cost ingredients.

3) Low hazards; DOT Class 1.4c.

4) Moderate regression rate: $\sim 0.1$ inches per second @ 200 psia.

In addition, the fuel had to exhibit efficient, stable combustion at operating pressures in the absence of external oxidizer. To this end, some emphasis was placed on minimizing soot and char production during the propellant fuel combustion. The approach chosen utilized the improved combustion characteristics of polyether (PPG) based binders with the conventional oxidizer, ammonium perchlorate (AP). Figure 1 illustrates the theoretical Isp performance of several options with $90 \%$ peroxide. This illustrates that fairly constant Isp performance is achieved at optimum stoichiometry for a fairly wide range of fuel types and AP loadings, albeit at substantially different oxidizer/fuel ratio's (O/F's). As AP content and/or PPG content increases, optimum O/F shifts to lower values.

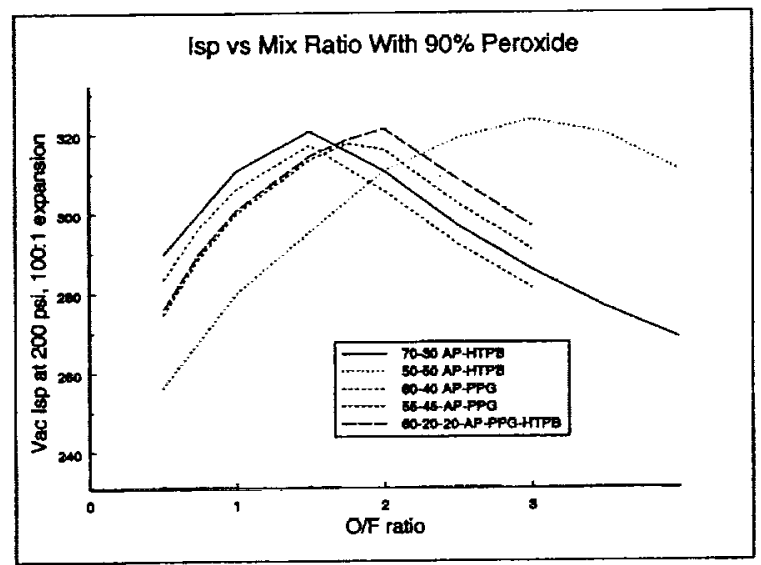

Figure 1. Theoretical Isp vs. mix ratio for several fuel variations with $90 \%$ hydrogen peroxide.

\section{Fuel Formulation and Testing}

Fuel formulation screening was conducted with cured strands, small ballistic test motors, and with a smallscale laboratory hybrid combustor operated with gaseous oxygen (GOX) or $90 \%$ hydrogen peroxide. AP was selected as the oxidizer. AP levels and particle size were varied. Binders examined included hydroxy terminated polybutadiene (HTPB), a commercial PPG, or mixtures of the two.
In general, it was found that compositions utilizing HTPB as the sole binder tended to produce large amounts of soot during combustion. This behavior led to concern over ultimate combustion efficiency with this approach. In addition, low pressure deflagration with HTPB based propellants tended to be very persistent, which is anticipated to interfere with extinguishment of the system. PPG tended to exhibit a higher pressure deflagration limit (PDL) than HTPB.

On the other hand, HTPB possesses a more favorable fuel value than $P P G$, providing improved Isp and higher optimum O/F. HTPB based formulations also provided much higher burn (regression) rates than PPG alone. A blended binder approach was, therefore, pursued as a compromise.

Figure 2 illustrates cured strand ballistic results for a typical formulation. The presence of the plateau is interesting in that it seemed to be characteristic of these fuel rich compositions and persisted in the small motor ballistic test results as well.

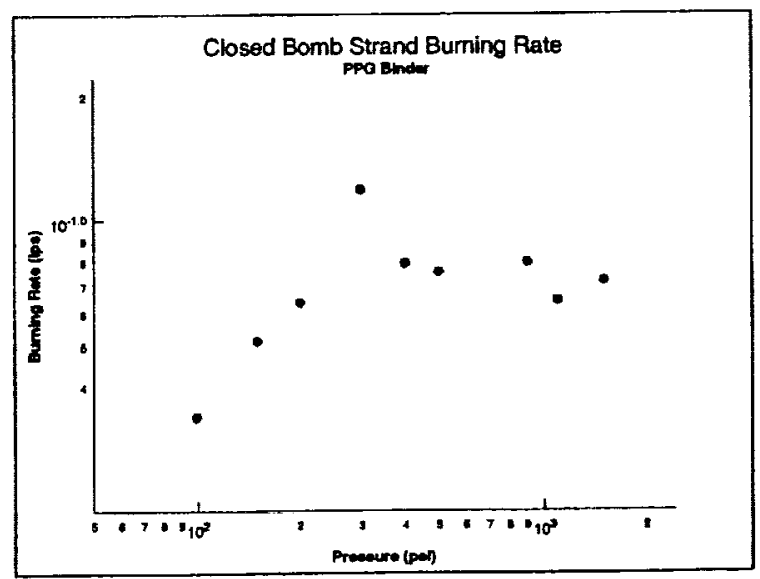

Figure 2. Closed bomb strand burning rates for PPG/AP gas generator propellant.

In general, obtaining ballistic data with these compositions proved challenging due to difficult ignition, extinguishment, and $L^{*}$ sensitivity. Typical test-to-test variability was found to be on the order of $15 \%$ to $20 \%$ in the absence of external oxidizer. During these experiments, it was found that AP particle size had a significant effect on ignition and sustained burning under these conditions. The presence of carbon black in the formulation also affected the ignition characteristics. Many of the fuel formulation candidates were also tested in a small-scale laboratory hybrid combustor based on the ballistic test motor. Grains were either a 1.5 -inch or 2-inch diameter center perforated (CP) configuration with almost identical port diameters. As illustrated in 
Figure 3, oxidizer (typically GOX) was injected into the forward end of the motor during operation.

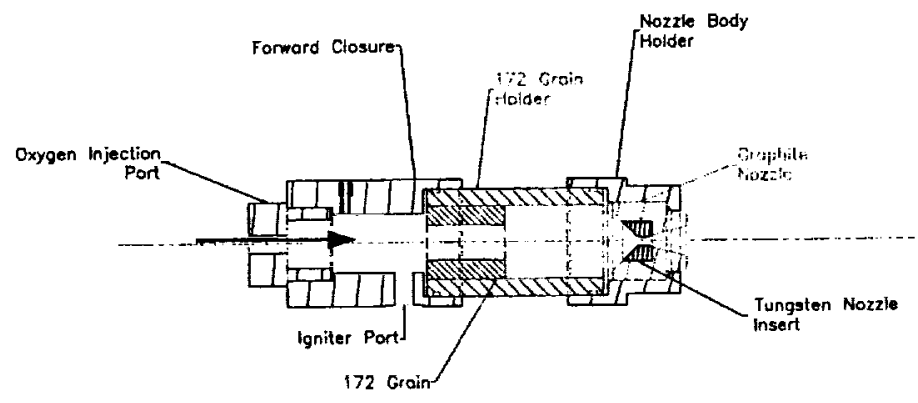

Figure 3. Schematic of 2-inch diameter lab-scale hybrid combustor.

Variables evaluated in this device consisted of oxidizer mass flow (flux), pressure and formulation. In these tests, ignition was accomplished by conventional squib, and the test terminated by oxidizer cut-off. Regression rates were determined by measuring the change in web thickness post-test or, alternatively, via weight loss. The majority of the testing with the $\mathrm{CP}$ configuration was done with the 1.5 -inch diameter motor, and the results indicated that, for most formulations tested, regression rate was very much governed by pressure with little if any oxidizer flux response noted as illustrated in Figure 4.

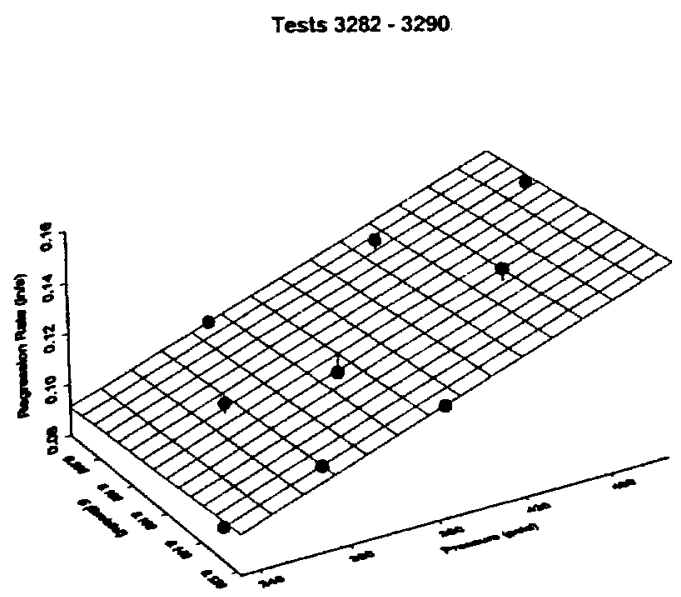

Figure 4. Regression rate vs. oxygen mass flux and chamber pressure for baseline fuel.

The (CP) grain configuration was operated with $90 \%$ hydrogen peroxide in addition to oxygen. In this case, a portable test stand and catalyst system manufactured by General Kinetics, LLC was interfaced with the combustor. Figure 5 is a photo of this system.

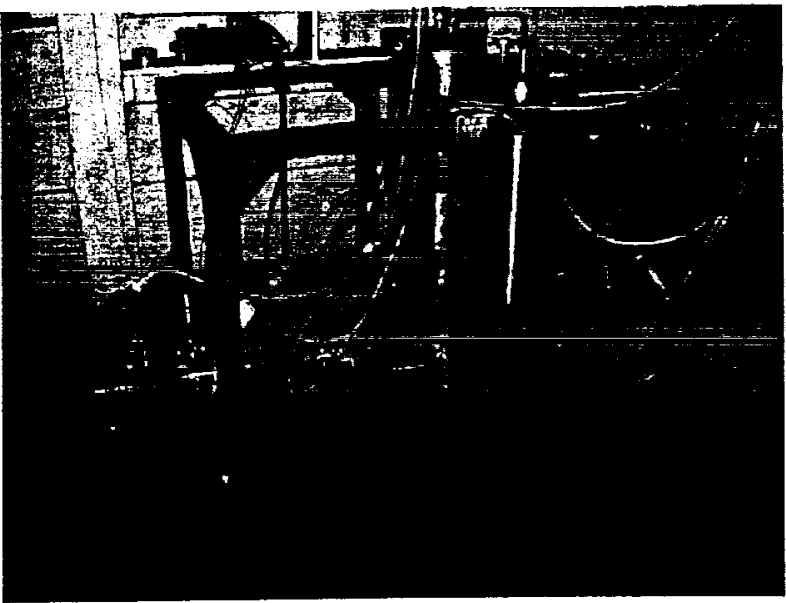

Figure 5. The hydrogen peroxide feed system and lab-scale combustor.

The 2-inch diameter $\mathrm{CP}$ grain configuration was utilized for the peroxide tests, principally to provide additional web for extended test times. Squib ignition was not necessary with the $\mathrm{CP}$ configuration since the hot, decomposed peroxide proved adequate for hypergolic ignition. Figure 6 is a reproduction of a typical CP test with this configuration showing the peroxide monopropellant operation phase and the grain ignition.

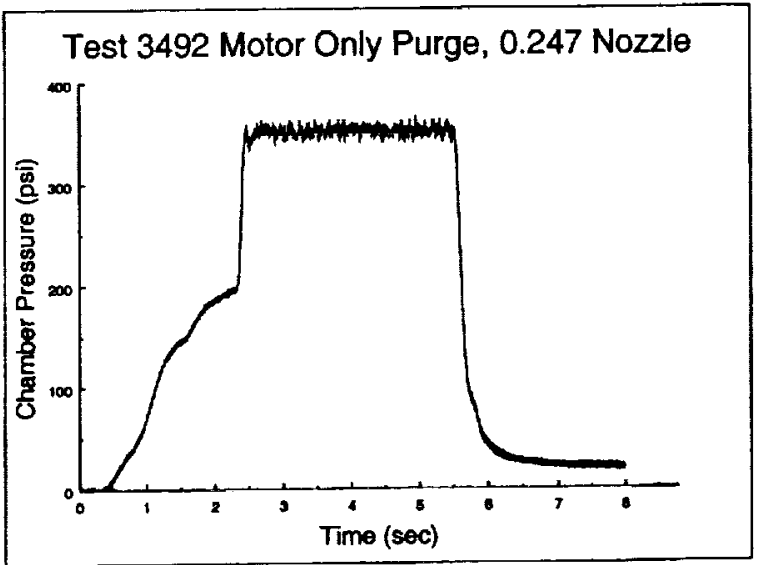

Figure 6. 2-inch CP test with hydrogen peroxide.

Operation was reasonably stable and the grain extinguished readily. The $O / F$ ratio for this test was 2.6 which is a little higher than stoichiometric (at roughly 2.0 ). In general, the regression rates measured with the peroxide were comparable to those obtained with GOX, particularly at lower pressures. Figure 7 compares regression rates as a function of pressure for the TU-628 (1.5-inch) and TU-172 (2-inch) motors with GOX and hydrogen peroxide respectively. 


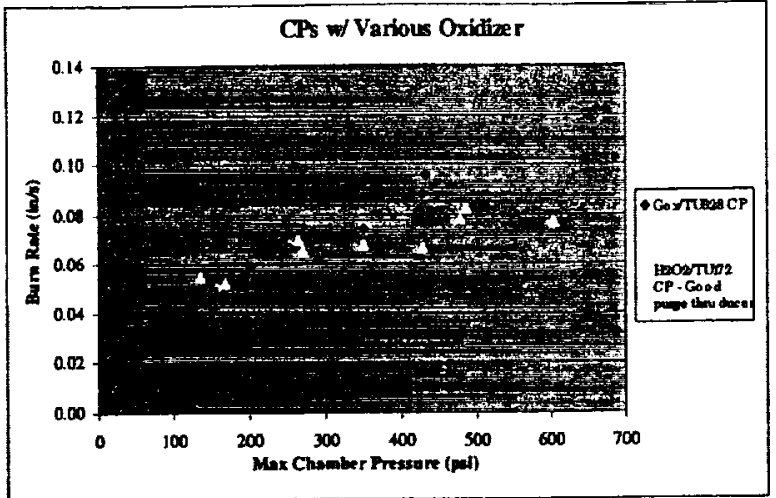

Figure 7. Gas Generator CP Performance with GOX and $\mathrm{H} 2 \mathrm{O} 2$.

Further experimentation revealed that the high pressure deviation observed in the plot was most likely due to the relative sizes of the motors and the presence of some velocity related flow effects inherent in the smaller 1.5 inch CP motor.

Development of an end-burning configuration for the lab-scale motor allowed further exploration with regard to flow environment. This motor, illustrated in Figure 8, utilized a 2-inch diameter grain and could be operated at a variety of length to diameter $(\mathrm{LD})$ and $\mathrm{L}^{*}$ (ratio of chamber volume divided by throat area) conditions by simply changing the length of the primary cylindrical combustion chamber. The end-burner combustor allowed flow effects during combustion to be varied by altering the angle of impingement of the injector port and the distance from injector to fuel grain surface. Environments ranging from cross-flow to directly impinging to minimal flow (static) were tested.

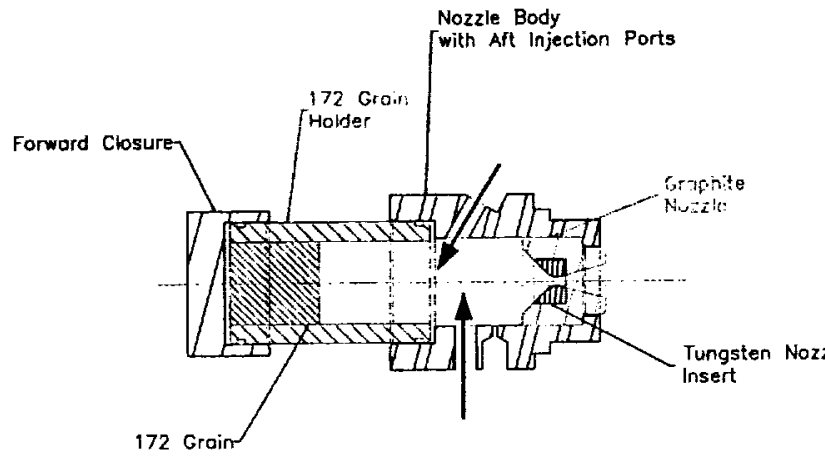

Figure 8. End burner configuration at moderate $L^{*}$ $(1000-2000$ in.) chamber length illustrating angled and radial flow geometry options.

As with the CP grain tests, both GOX and $90 \%$ hydrogen peroxide were used for fuel testing in the end burner.
The results from these tests led to a few surprises. As shown by the pressure plots in Figures 9 and 10 , combustion chamber $L / D$, and therefore $L^{*}$, had a significant effect on combustion performance with some fuels. Both tests shown were conducted with identical fuel, GOX mass flow rate and throat area, differing only in length of the combustion chamber ( 3.5 inches versus

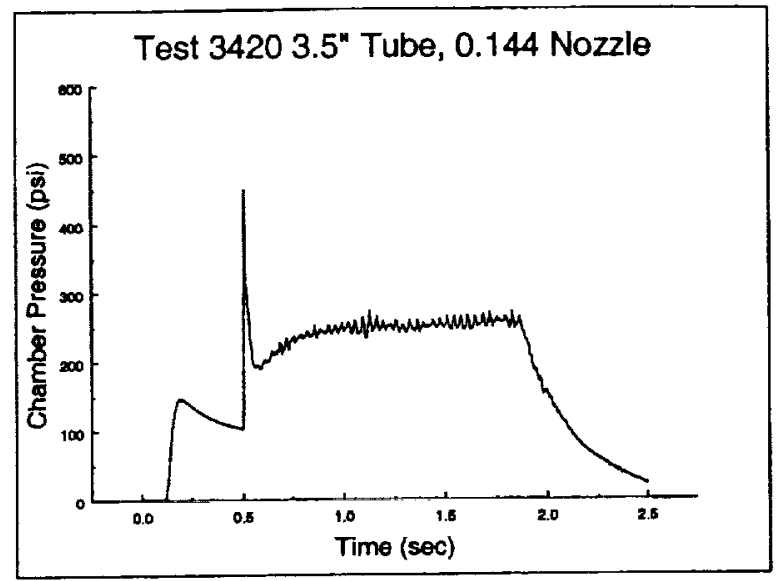

7.5 inches).

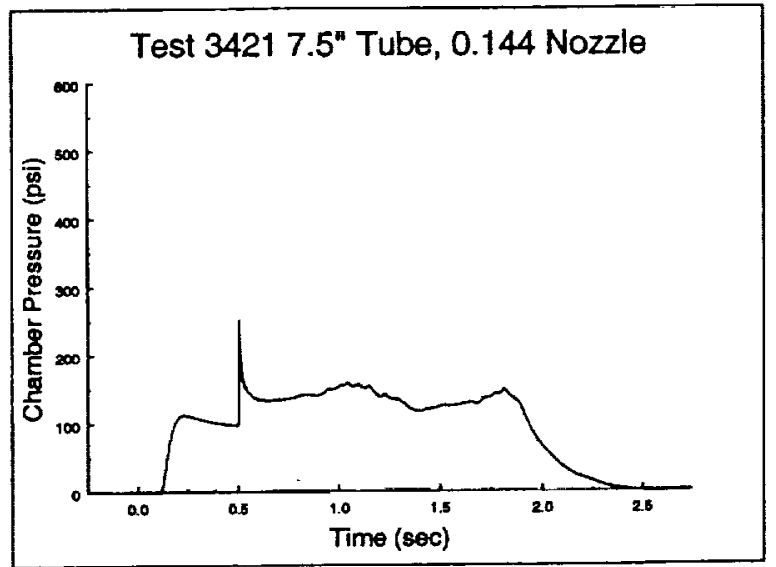

Figures 9 and 10. Pressure vs. time for end burner at 3.5 inch and 7.5 inch combustion chamber lengths. Pressure spike at 0.5 seconds is from the squib.

Based on these results, it became apparent that some formulations that performed well in a CP cross-flow environment were unduly sensitive in the more staticflow end burner environment. Not only were the chamber pressures reduced at large $L D$, but regression rates were halved as well. This observation implied that the combustion was not stable under conditions of very low oxidizer flow, completely unacceptable for proper 
motor operation. Fortunately, the formulation responded well to an AP size adjustment, which proved to be an effective remedy. As shown in Figure 11 (compared to Figure 9), identical motor pressure and regression rates were achieved at both $L D$ conditions with the modified fuel. The final formulation was designated DL-H485 and was further characterized in the end burner with $90 \%$ hydrogen peroxide.

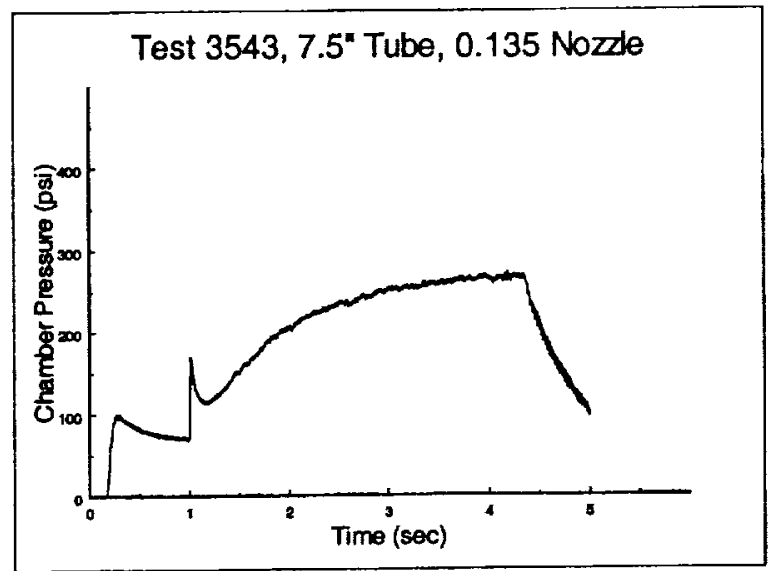

Figure 11. Long LJD GOX test with improved formulation.

The lack of significant oxidizer cross-flow or impingement on the fuel grain surface led to dramatically increased ignition delays with $\mathrm{H} 2 \mathrm{O} 2$ relative to those observed with the $\mathrm{CP}$ tests. Consequently, squib ignition was required for these tests, coupled with adequate delay time for sufficient heating of the hardware (typically 15 seconds).

Regression rates obtained with the baseline fuel with both GOX and peroxide in the end burner are compared in Figure 12. In this case, the regression rates are again very similar with those from the peroxide tests being slightly higher than with the GOX. This is suggested to be due to the fact that the O/F ratios for all of the tests was roughly 1.5 to 3 , which is fairly close to optimum for $90 \%$ peroxide but considerably oxidizer rich for the GOX tests. Since this equates to lower flame temperature, it is not unreasonable to expect some effect on observed regression rate. Overall, a regression rate of about 0.06 inches per second at $200 \mathrm{psi}$ with a pressure exponent of 0.3 was obtained for the DL-H485 fuel and $90 \%$ hydrogen peroxide.

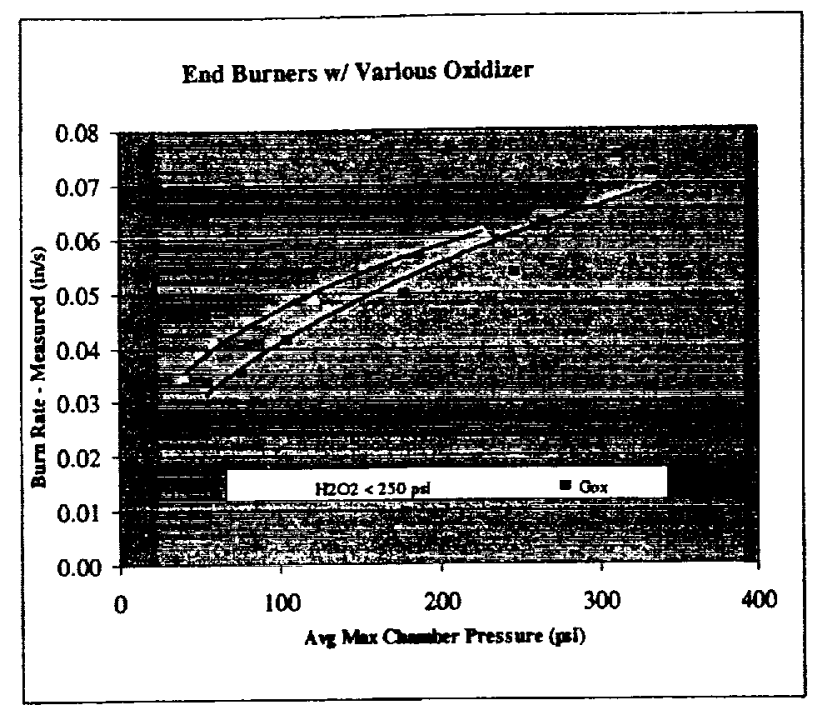

Figure 12. Regression rate comparison for DL-H485 fuel end burner with GOX and $90 \%$ peroxide.

Variation of the oxidizer injection angle and distance to fuel surface were performed to confirm the sensitivity of the fuel to flow effects at various extremes. As shown in figure 13 , an increase in regression rate was observed when impinging flow prevailed in the combustor. These regression rates are similar to what was observed in the $\mathrm{CP}$ tests and also tends to imply that the fuel is sensitive to flow (flux) effects. This may appear contrary to the data presented in Figure 4 which suggests minor sensitivity over the ranges tested. These ranges represent modest velocity conditions (all at fairly high oxygen mass flux) as opposed to the conditions in the end burner. Under these conditions, the effects between a direct impinging flow and a non-impinging condition are expected to be considerably greater.

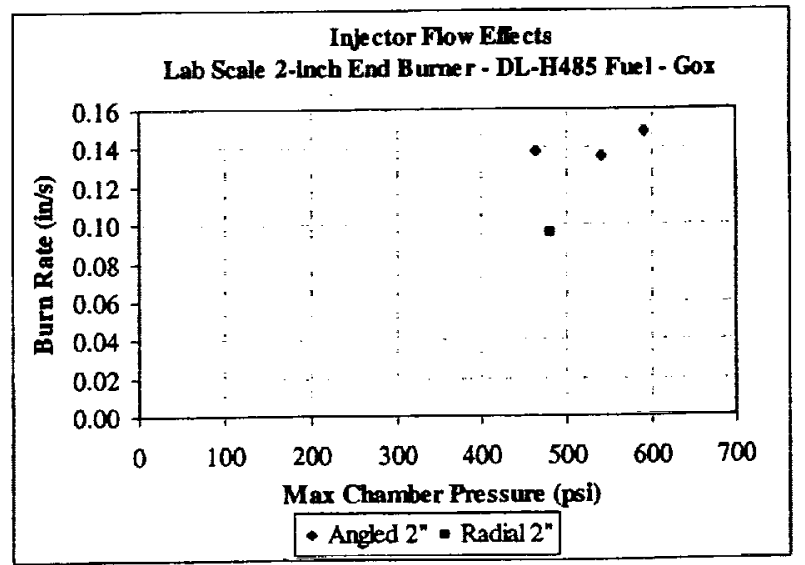

Figure 13. Lab-scale Injector Flow Effects. 


\section{$\underline{\text { Scale-Up }}$}

DL-H485 was successfully scaled up to the 5-gallon mix level $(50 \mathrm{lb}$.) to support 11 -inch and 24 -inch diameter static motor testing. Grains were fabricated as end burners by casting into a lined silica-phenolic "cup" and cured. These were then cartridge loaded into the respective test hardware for testing. Figure 14 is a photograph of a representative 11 -inch fuel grain.

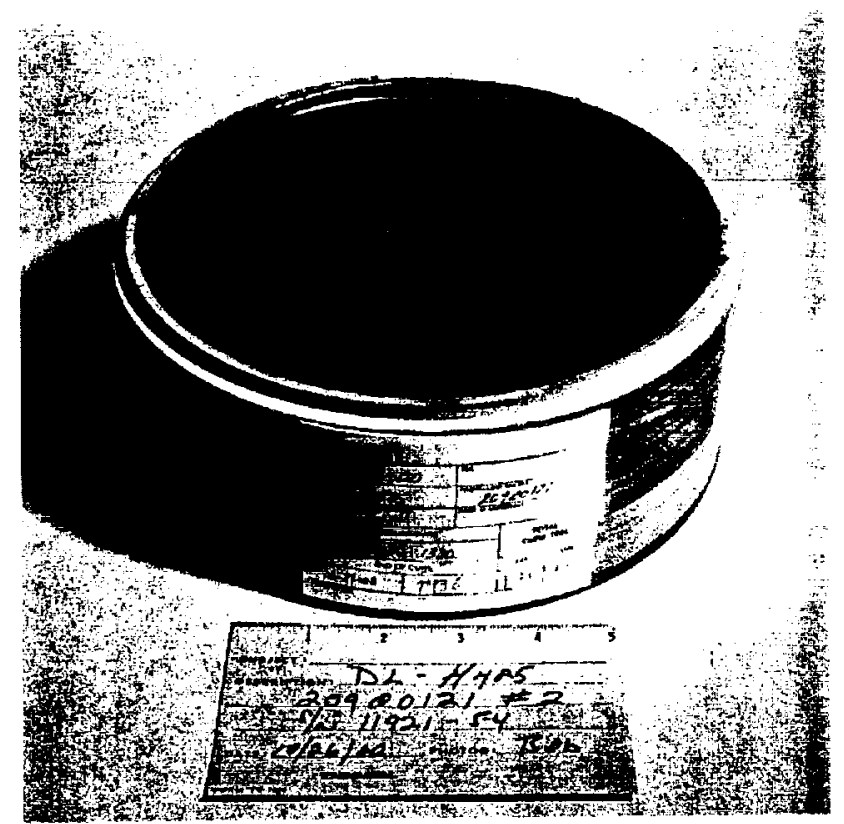

Figure 14. As-cast 11-inch solid fuel grain.

Testing of the 11-inch and 24-inch motors was conducted at Stennis Space Flight Center with $90 \%$ hydrogen peroxide. Test operating conditions were similar to those employed in the lab-scale studies with respect to test times, pressures, O/F and L*. The 11 - and 24-inch test results agreed closely with the lab-scale results discussed herein.

Hazard testing of DL-H485 resulted in it's being granted a DOT Class $1.4 \mathrm{c}$ hazards classification which has significant ramifications for manufacture, shipping, storage and handling. This allows the gas generator cycle hybrid motor as developed here to retain one of the more significant advantages of classical hybrid designs - that of reduced handling and storage hazards relative to conventional propellants.

\section{Summary and Conclusions}

A gas generating fuel formulation (DL-H485) has been developed which meets the goals of the program, i.e., theoretical Isp $>310$ seconds, low cost ingredients, low hazards, moderate regression rate, self-deflagrating, stable and efficient combustion in both low and high $\mathrm{L}^{*}$ configurations, and which extinguishes on oxidizer shutoff.

The lab-scale combustor has proved to be invaluable in the development of gas generator hybrid fuel formulations. The results from lab-scale testing closely agree with the larger 11 -inch and 24-inch motors indicating excellent scale-up characteristics. This is obviously quite advantageous for future hybrid fuel and motor development efforts.

Fuel formulation and testing to date have demonstrated the viability of a peroxide hybrid upper stage motor system. 